\title{
MicroRNA-382 inhibits cancer cell growth and metastasis in NSCLC via targeting LMO3
}

\author{
DINGZHU CHEN ${ }^{1}$, YI ZHANG ${ }^{1}$, YONG LIN ${ }^{1}$, FEIMIN SHEN $^{1}$, ZHIJIAN ZHANG ${ }^{2}$ and JIGUANG ZHOU $^{3}$ \\ Departments of ${ }^{1}$ Cardiac and Thoracic Surgery, ${ }^{2}$ Clinical Laboratory and ${ }^{3}$ Hospital Information, \\ Zhangzhou Hospital Affiliated to Fujian Medical University, Zhangzhou, Fujian 363000, P.R. China
}

Received February 7, 2018; Accepted May 25, 2018

DOI: 10.3892/etm.2019.7271

\begin{abstract}
Recent studies have revealed a pivotal role of microRNAs (miRs) in regulating the initiation and development of multiple types of cancer. In the present study, it was discovered that miR-382 may be an important tumor suppressor in non-small cell lung cancer (NSCLC). It was demonstrated that miR-382 expression was downregulated in tumor tissues from patients with NSCLC compared with adjacent normal tissues. Furthermore, overexpression of miR-382 suppressed cell proliferation and cell migration of NSCLC cells. In addition, reverse transcription-quantitative polymerase chain reaction and the luciferase reporter assay revealed that LIM-only protein 3 (LMO3), an oncogene, acted as a direct target gene of miR-382. Notably, overexpression of miR-382 did not alter cell proliferation or migration in LMO3-silenced A549 cells. Furthermore, analysis of patient tissues indicated an elevation of LMO3 expression in tumor tissues compared with adjacent normal tissues and a negative association between miR-382 and LMO3 mRNA expression levels. Taken together, the present findings indicated that miR-382 inhibited NSCLC cell proliferation and metastasis by targeting LMO3, suggesting a tumor suppressor role of miR-382 in NSCLC.
\end{abstract}

\section{Introduction}

Lung cancer is the leading cause of cancer-associated mortality in both men and women worldwide, and $>80 \%$ of lung cancer cases are diagnosed as non-small cell lung cancer (NSCLC) (1). Although great achievements have been made in improving NSCLC diagnosis and therapeutic approaches, including the development of targeted therapies, the overall survival rate remains very low (with a 5-year survival rate of $\sim 15 \%$ ), which is accompanied with a high recurrence rate (2).

Correspondence to: Dr Dingzhu Chen, Department of Cardiac and Thoracic Surgery, Zhangzhou Hospital Affiliated to Fujian Medical University, 59 Shengli Road, Zhangzhou, Fujian 363000, P.R. China E-mail: chendingzhufjmu@sohu.com

Key words: micorRNA-382, LIM-only protein 3, non-small cell lung cancer, cell growth and metastasis
Thus, it is of importance to uncover the molecular mechanism of cancer cell proliferation and metastasis in NSCLC to facilitate the development of novel drugs.

microRNAs (miRNAs or miRs) are small, non-coding RNAs that function by binding to the 3'untranslated regions (3'UTRs) of target mRNAs to induce mRNA degradation or suppression of transcription (3). miRNAs have been reported to be associated with cancer development via targeting oncogenes or tumor suppressors in various cancer types, including NSCLC (4-7). However, the role of miR-382 in NSCLC development remains to be fully elucidated.

LIM-only protein 3 (LMO3) was initially discovered based on the sequence homology with LMO1 (8). As a member of the LMO family, LMO3 is an important regulator of cell growth and differentiation during embryonic development (9). Later research revealed that LMO3 acts as an oncogene through interacting with nescient helix-loop-helix 2 in neuroblastoma (10). In NSCLC, LMO3 was previously reported to promote cancer cell proliferation and metastasis, and was negatively regulated by miR-630 (11).

In the present study, a decrease in miR-382 expression was observed in the tumor tissues of patients with NSCLC compared with adjacent normal tissues. Additionally, overexpression of miR-382 significantly inhibited NSCLC cell growth and migration. Furthermore, elevated miR-382 expression decreased LMO3 mRNA and protein expression levels. The luciferase assay confirmed that LMO3 was a direct target of miR-382. More importantly, in LMO3-silenced A549 cells, miR-382 mimics did not exhibit anti-cancer effects. A negative association was also identified between LMO3 and miR-382 expression in tumor tissues from patients with NSCLC. Taken together, the present findings demonstrated a tumor suppressor role of miR-382 in NSCLC.

\section{Materials and methods}

Patient samples. A total of 30 tumor tissues and matched adjacent normal tissues were collected from 30 patients (10 males and 20 females; age range, 31-68 years) who had undergone surgery to treat NSCLC at the Zhangzhou Hospital Affiliated to Fujian Medical University (Zhangzhou, China) between January 2014 and January 2015. Fresh tissue samples were confirmed via histopathological examination and then immediately stored in a $-80^{\circ} \mathrm{C}$ refrigerator prior to subsequent 
experimentation. Patients were graded and the tumor sizes and the metastatic activity were assessed according to American Joint Committee on Cancer Staging V7 (12). Diagnosis was confirmed by pathological analysis. The patients included in the present study did not receive radiation or chemotherapy prior to surgery. All patients provided written informed consent and the Ethics Committee of Zhangzhou Hospital Affiliated to Fujian Medical University approved the present study.

Cell culture. The normal human bronchial epithelial cell line BEAS-2B, 293 cells, and human lung cancer cell lines, H1299, H23 and A549 were purchased from the American Type Culture Collection (Manassas, VA, USA). All cell lines were maintained in Dulbecco's modified Eagle's medium (Gibco; Thermo Fisher Scientific, Inc., Waltham, MA, USA) supplemented with $10 \%$ fetal bovine serum (FBS; Hyclone; GE Healthcare Life Sciences, Logan, UT, USA) and 1\% penicillin-streptomycin solution (Gibco; Thermo Fisher Scientific, Inc.) in a humidified atmosphere containing $5 \% \mathrm{CO}_{2}$ at $37^{\circ} \mathrm{C}$.

Target prediction for miRNAs. Bioinformatics prediction of the target genes of miRNA and miRNA binding sites was performed using the online database, miRanda (http://www. microrna.org/). LMO3 was identified as a target gene of miR-382 and was verified by experimental analysis.

Plasmid construction. The LMO3 3'UTR was amplified from the cDNA of A549 cells using PrimeSTAR ${ }^{\circledR}$ Max DNA Polymerase (Takara Bio, Inc., Otsu, Japan) and cloned into pGL3 plasmids (Invitrogen; Thermo Fisher Scientific, Inc.) to construct pGL3-LMO3 3'UTR-wild type (WT). The LMO3 primer sequences were as follows: Forward: 5'-GCTCTA GAAACACTTTAAGTTTGATGTG-3'; Reverse: 5'-GCTCTA GATAAGATCCTGACAATTATAAT-3'. The thermocycling conditions were as follows: Denaturation at $98^{\circ} \mathrm{C}$ for $10 \mathrm{sec}$; annealing at $55^{\circ} \mathrm{C}$ for $10 \mathrm{sec}$; elongation at $72^{\circ} \mathrm{C}$ for $60 \mathrm{sec}$ for 35 cycles. A549 cDNA was prepared from extracted RNA using the TRIzol reagent (Invitrogen; Thermo Fisher Scientific, Inc.). Samples were the reverse-transcribed into cDNA using the RevertAid RT Reverse Transcription kit (Thermo Fisher Scientific, Inc.) according to the manufacturer's protocol. pGL3-LMO 3'UTR-mutant (mut) was constructed using a QuikChange Site-Directed Mutagenesis kit (Agilent Technologies, Inc., Santa Clara, CA, USA), according to the manufacturer's protocol.

Small interfering (si)RNA transfection. LMO3 siRNAs and control siRNA were purchased from GE Healthcare Dharmacon, Inc., Lafayette, CO, USA. Knockdown of LMO3 was achieved by transfection of LMO3-specific siRNAs into A549 cells using Lipofectamine RNAiMAX (Invitrogen; Thermo Fisher Scientific, Inc.). Briefly, 30 pmol LMO3 siRNAs or control siRNA were mixed with Lipofectamine RNAiMAX in OptiMEM (Invitrogen; Thermo Fisher Scientific, Inc.), incubated for $15 \mathrm{~min}$ at $37^{\circ} \mathrm{C}$ and subsequently added into each well containing culture medium in 6 -well or 96 -well plates. Cells were cultured for a further $48 \mathrm{~h}$ prior to experimentation. The siRNA sequences were as follows: LMO3 siRNA1, CGU CCCUACUCGAAUGAUG; LMO3 siRNA2, CCGUUACAC CAAAGAGCCUC; LMO3 siRNA3, GUAGUCCGUCUG
GCAAAGGA; control siRNA, UAAGGCUAUGAAGAG AUAC.

Luciferase assay. miR-382 mimics and miR-negative control (NC) mimics were synthesized and purchased from Guangzhou RiboBio Co., Ltd. (Guangzhou, China). The dual luciferase assay was performed using a Dual-Luciferase ${ }^{\circledR}$ Reporter Assay System (Promega Corporation, Madison, WI, USA). Briefly, 293 cells were cultured in 24-well plates and cotransfected with miR-382 mimics or miR-NC mimics, pGL3-LMO3 3'UTR-WT or pGL3-LMO3 3'UTR-mut and Renilla luciferase vector using Lipofectamine 2000 (Invitrogen; Thermo Fisher Scientific, Inc.). Following 48 h, cells were collected and the dual-luciferase activity was examined with Renilla luciferase as the internal control. The sequences were of the miRs were as follows: miR-382 mimic, 5'GAAGUUGUUCGUGGUGGA UUCG3' and miR-NC mimic, 5'CAUGUAGUACGCGUU GAGUACC3'.

Western blot analysis. Anti-LMO3 antibody (cat. no 517019, 1:1,000) was purchased from Santa Cruz Biotechnology, Inc. (Dallas, TX, USA) and anti-GAPDH antibody (cat. no G8795; 1:5,000) was obtained from Sigma-Aldrich; Merck KGaA (Darmstadt, Germany). Cell lysates were prepared using radioimmunoprecipitation assay lysis buffer (Beyotime Institute of Biotechnology, Haimen, China). The protein concentration was determined using a Pierce BCA Protein Assay kit (Thermo Fisher Scientific, Inc.). Proteins $(20 \mu \mathrm{g})$ were separated using $8 \%$ SDS-PAGE and transferred to polyvinylidene difluoride membranes. Membranes were subsequently blocked with 5\% non-fat milk at room temperature for $1 \mathrm{~h}$ and incubated with the indicated primary antibodies $(1: 1,000)$ overnight at $4^{\circ} \mathrm{C}$. The next day, membranes were washed with TBS-Tween 20 and incubated with horseradish peroxidase-conjugated secondary antibody for $1 \mathrm{~h}$ at room temperature (cat. no. 516102; Santa Cruz Biotechnology, Inc.; 1:10,000). Subsequently, the membranes were developed using ECL Prime Western Blotting Detection Reagents (GE Healthcare Life Sciences). Images were captured and analyzed using ImageQuant TL 7.0 (GE Healthcare Life Sciences). GAPDH served as a loading control.

Reverse transcription-quantitative polymerase chain reaction $(R T-q P C R)$. An miRNeasy Mini Kit (Qiagen, Inc., Valencia, CA, USA) was used to extract total RNA from patient tissue and cells (BEAS-2B, 293, H1299, H23 and A549) in accordance with the manufacturer's instructions. Following this, a NanoDrop 2000 kit (Thermo Fisher Scientific, Inc.) was used to measure the concentration and quality of RNA. TransScript First-Strand cDNA Synthesis SuperMix (Beijing Transgen Biotech Co., Ltd., Beijing, China) was used to reverse transcribe RNA into cDNA following manufacturer's protocol. qPCR was performed with a CFX96 Touch $^{\text {TM }}$ Real-Time PCR Detection System (Bio-Rad Laboratories, Inc., Hercules, CA, USA) using SYBR Premix Ex Taq (Takara Bio, Inc.). The thermocycling conditions were as follows: Pre-denaturation at $95^{\circ} \mathrm{C}$ for $30 \mathrm{sec}$; denaturation at $95^{\circ} \mathrm{C}$ for $5 \mathrm{sec}$; annealing and elongation at $60^{\circ} \mathrm{C}$ for $30 \mathrm{sec}$ for 40 cycles. The relative expression of genes was calculated using the $2^{-\Delta \Delta \mathrm{Cq}}$ method (13). U6 and GAPDH were used as internal controls for miRNA and 
A

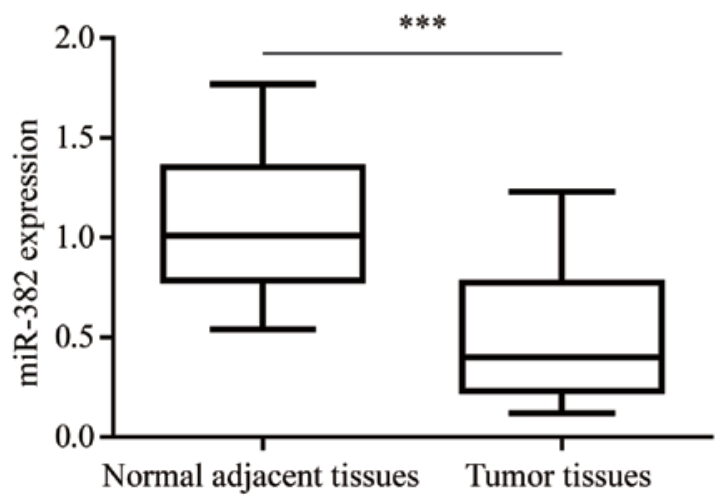

B

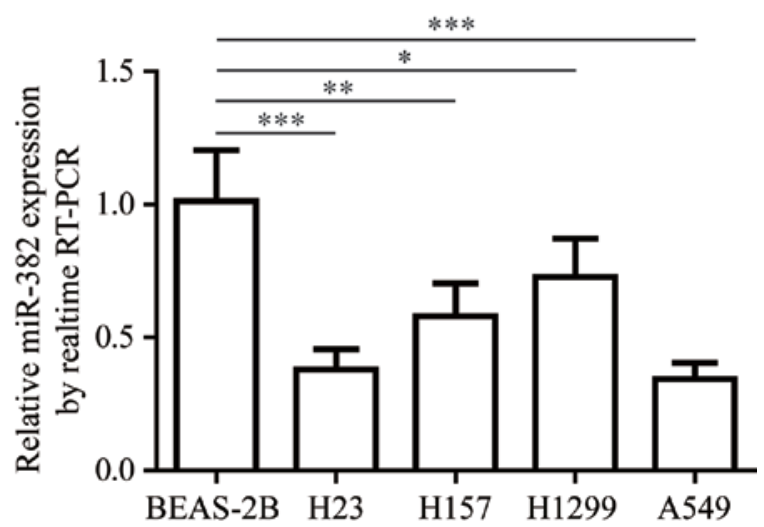

Figure 1. miR-382 expression was decreased in tumor tissues from patients with NSCLC and NSCLC cell lines. (A) RT-qPCR was used to measure miR-382 expression levels in tumor tissues and matched normal adjacent tissues from 30 patients with NSCLC. (B) Comparison of miR-382 expression levels in the human bronchial epithelial cell line, BEAS-2B, with that in the human lung cancer cell lines $\mathrm{H} 1299, \mathrm{H} 23$ and $\mathrm{A} 549$. ${ }^{*} \mathrm{P}<0.05{ }^{*}{ }^{* *} \mathrm{P}<0.01$; ${ }^{* * *} \mathrm{P}<0.0001$. miR, microRNA; NSCLC, non-small cell lung cancer; RT-qPCR, reverse transcription-quantitative polymerase chain reaction.

mRNA, respectively. Sequences for primers used were as follows: miR-382, forward 5'-CTGCAATCATTCACGGAC AAC-3' and reverse 5'-GTGTCGTCGAGTCGGCAATTC-3'; LMO3, forward 5'-ATGCTCTCAGTCCAGCCAGA-3' and reverse 5'-TCAGCGAACCTGGGGTGCAT-3'; U6, forward 5'-CCTGCTTCGGCAGCACA-3' and reverse 5'-TGGAAC GCTTCACGAA-3'; and GAPDH, forward 5'-CCACTCCTC CACCTTTGAC-3' and reverse 5'-ACCCTGTTGCTGTAG CCA-3'.

Cell proliferation assay. Cell growth was measured using a Cell Counting Kit (CCK)-8 (Dojindo Molecular Technologies, Inc., Kumamoto, Japan) according to the manufacturer's protocol. Briefly, cells were seeded in 96 -well plates at $37^{\circ} \mathrm{C}$. On the following day, $10 \mu \mathrm{l}$ CCK-8 solution was added into each well and the cells were incubated for $2 \mathrm{~h}$ at $37^{\circ} \mathrm{C}$. The absorbance at $450 \mathrm{~nm}$ was detected using a microplate reader (Bio-Rad Laboratories, Inc.). At 24, 48 and $72 \mathrm{~h}$ following transfection with miR-382 mimics or miR-NC mimics, the cell number was analyzed using CCK-8.

Wound-healing assay. Cell migration ability was measured using a wound-healing assay. A549 cells were cultured in 6 -well plates at $37^{\circ} \mathrm{C}$. On the following day, a wound was made by introducing a scratch at the center of each well with a $10-\mu 1$ pipette tip. Culture medium was replaced with fresh medium containing $1 \%$ FBS, and the A549 cells were cotransfected with miR-382 mimics or miR-NC mimics and LMO3 siRNA or control siRNA using the protocol as aforementioned in the luciferase assay. An image of the scratch was then captured. Following $40 \mathrm{~h}$, a second image of the scratch was captured. Subsequently, the percentage of migratory cells was analyzed using Image Pro Plus 6 (Media Cybernetics, Inc., Rockville, MD, USA).

Statistical analysis. All statistical analyses were performed using GraphPad Prism 5.0 software (GraphPad Software, Inc., La Jolla, CA, USA). Data are presented as the mean \pm standard deviation. Differences between two groups were analyzed using Student's t-test and multiple groups were analyzed using one-way analysis of variance followed by the Newman-Keuls post hoc test. $\mathrm{P}<0.05$ was considered to indicate a statistically significant difference.

\section{Results}

miR-382 expression is reduced in tumor tissues from patients with NSCLC and NSCLC cell lines. To explore the role of miR-382 during NSCLC cancer progression, the miR-382 expression levels between tumor tissues and normal adjacent tissues from 30 patients with NSCLC were compared. RT-qPCR detected a significant decrease in miR-382 expression in tumor tissues compared with normal adjacent tissues (Fig. 1A). In addition, significantly reduced miR-382 expression levels were observed in 3 NSCLC cell lines compared with BEAS-2B, a normal human bronchial epithelial cell line (Fig. 1B).

miR-382 suppresses cell proliferation and migration in A549 cells. To further investigate how miR-382 influences NSCLC cell behavior, the cell proliferation and migration abilities of A549 cells were evaluated following the overexpression of miR-382 using miR-382 mimics. Compared with the control and miR-NC mimics, miR-382 mimics significantly increased the miR-382 expression levels in A549 cells (Fig. 2A). Notably, overexpression of miR-382 provoked an inhibitory effect on A549 cell proliferation and migration (Fig. 2B-D), suggesting that miR-382 may suppress NSCLC cell growth and metastasis.

LMO3 is a target gene of miR-382. LMO3 has been reported to promote NSCLC growth and metastasis (11). miRanda analysis indicated that LMO3 was a potential target gene of miR-382 (Fig. 3A). In A549 cells, overexpression of miR-382 decreased LMO3 mRNA and protein expression levels (Fig. 3B and C). Subsequently, whether LMO3 was a direct target gene of miR-382 was investigated using the dual luciferase assay. The results demonstrated that miR-382 mimics significantly reduced the luciferase activity in 293 cells transfected with LMO3 3'UTR-WT compared with transfection of miR-NC mimics (Fig. 3D). In addition, miR-382 overexpression did not alter the luciferase activity of 293 cells transfected with LMO3 3'UTR-Mut (Fig. 3D). 
A

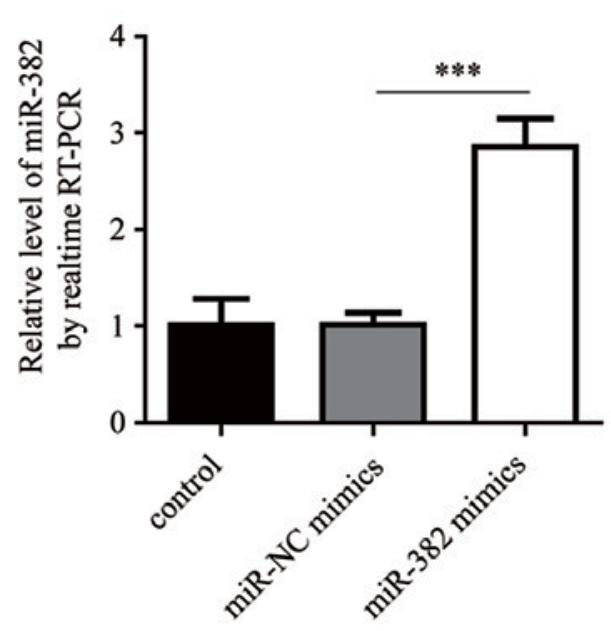

C
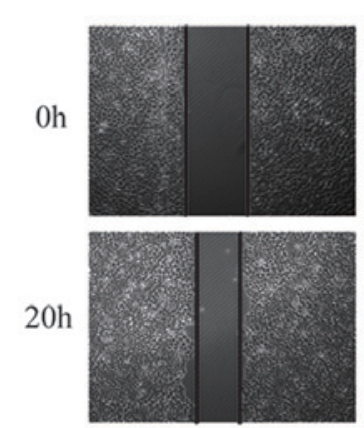

control
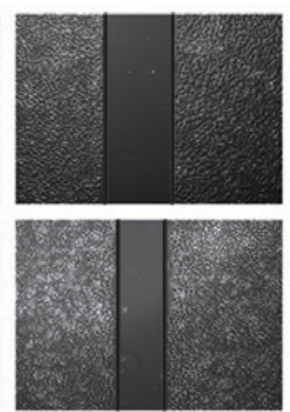

miR-NC mimics
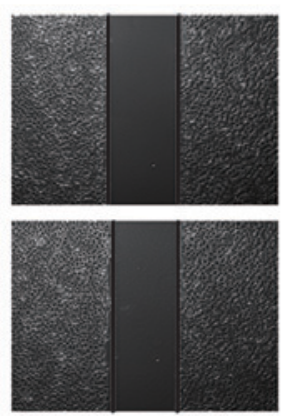

miR-382 mimics

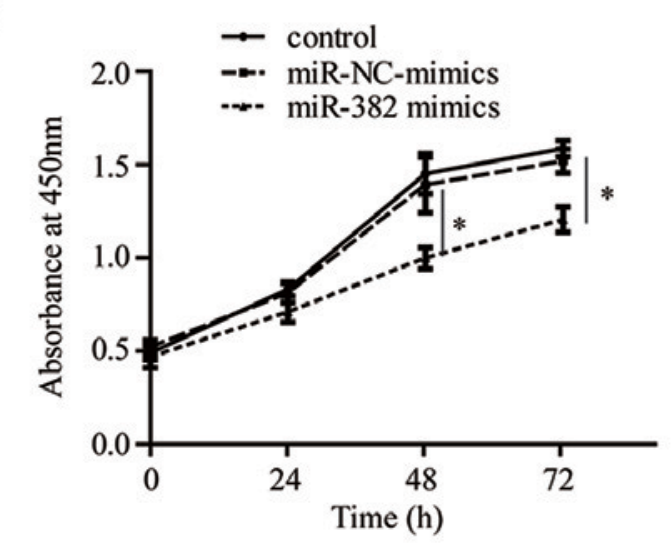

D

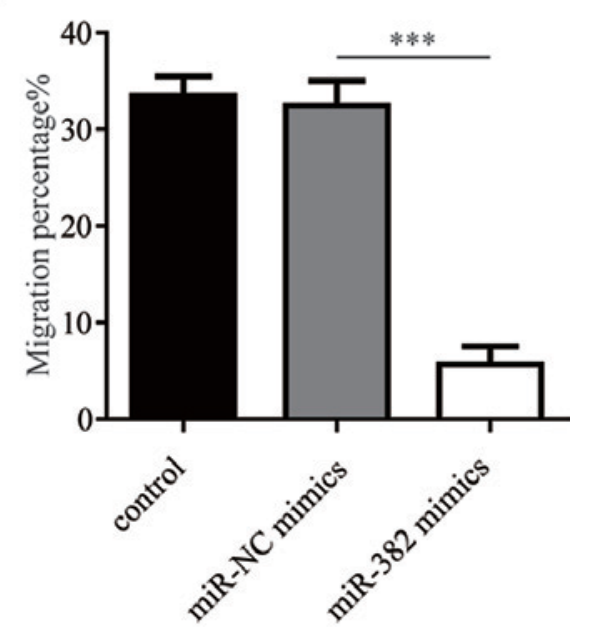

Figure 2. miR-382 inhibited A549 cell proliferation and migration. (A) Compared with the control and miR-NC mimics groups, miR-382 mimics significantly increased miR-382 expression levels. (B) miR-382 mimics suppressed A549 cell proliferation in comparison with the control and miR-NC mimics groups. (C) miR-382 mimics suppressed A549 cell migration in comparison with the control and miR-NC mimics groups (magnification, x40). (D) Quantitative analysis of $(\mathrm{C}) .{ }^{*} \mathrm{P}<0.01 ;{ }^{* * * *} \mathrm{P}<0.0001$. miR, microRNA; NC, negative control; RT-qPCR, reverse transcription-quantitative polymerase chain reaction.

miR-382 inhibits A549 cell proliferation and migration via LMO3. To further investigate whether miR-382 is associated with the regulation of A549 cell proliferation and migration, A549-cell proliferation and migration abilities were detected following combining miR-382 overexpression with LMO3-knockdown. In A549 cells, decreased LMO3 protein expression levels were observed following transfection with LMO3 siRNA1, LMO3 siRNA2 or LMO3 siRNA3 (Fig. 4A). LMO3 siRNA1 exhibited the highest efficiency in regulating LMO3 protein expression levels and was therefore used for the following experiments. Results indicated that silencing LMO3 markedly reduced A549 cell proliferation ability; however, combined LMO3 knockdown and miR-382 overexpression did not significantly induce further cell growth inhibition (Fig. 4B). Furthermore, compared with the LMO3 knockdown group, there was no significant difference in the cell migration ability in cells with combined LMO3 silencing and miR-382 overexpression (Fig. 4C and D). These data indicated that, miR-382 did not show significant effects on the cell proliferation and cell migration of A549 cells without LMO3, suggesting that miR-382 may primarily rely on the negative regulation of LMO3 to suppress NSCLC cell growth and migration.
LMO3 is overexpressed and negatively associated with miR-382 in tumor tissues from patients with NSCLC. LMO3 expression levels in patients with NSCLC were investigated. Significant elevation of LMO3 mRNA expression levels were detected in tumor tissues compared with normal adjacent tissues in patients with NSCLC (Fig. 5A). Additionally, via analysis of the miR-382 and LMO3 mRNA expression levels in NSCLC tumor tissues, a significant negative association between miR-382 and LMO3 mRNA expression levels was revealed (Fig. 5B). Therefore, miR-382 may promote cancer cell growth and metastasis via targeting LMO3 in patients with NSCLC.

Expression of miR-382 and LMO3 in lung carcinoma tissues. The fold changes of miRNA-382 and LMO3 were tested in the 30 patients in the present study (10 male and 20 female) with respect to various patient characteristics, including sex, age, tumor size, histological grade and lymph node metastasis. As presented in Table I, there was no significant difference in miRNA-382 expression according to sex $(\mathrm{P}=0.938)$, age $(\mathrm{P}=0.331)$ or histological grade $(\mathrm{P}=0.156)$; however, low miRNA-382 expression was significantly associated with 
A
LMO3 3'UTR (Position 1829-1836)

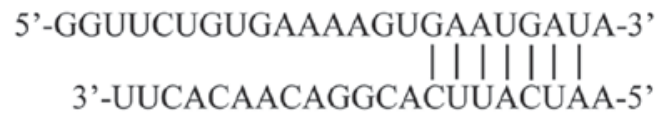
miR-382

C

$\mathrm{B}$

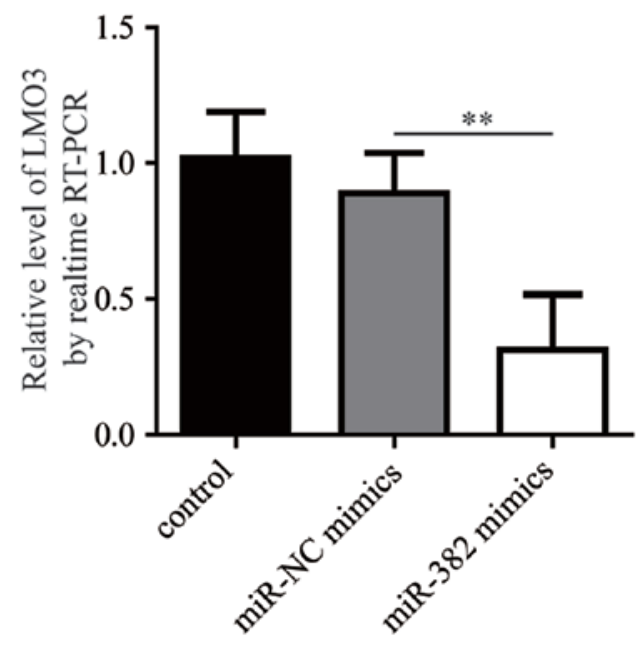

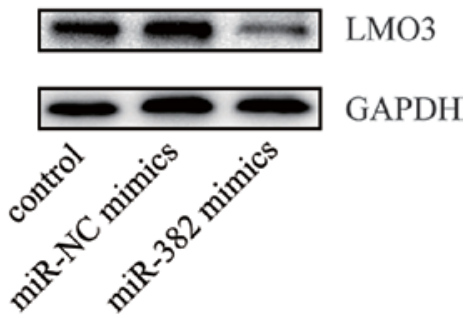

D

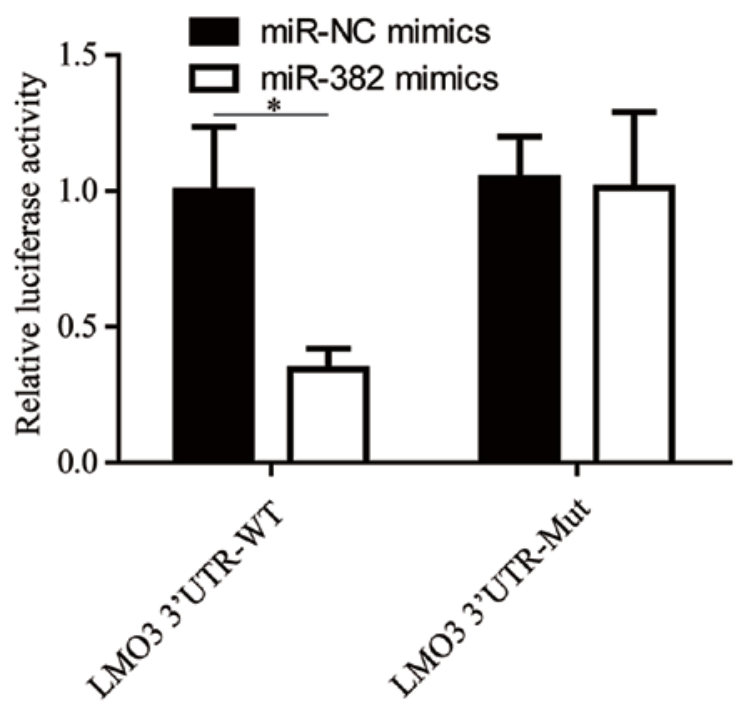

Figure 3. LMO3 is a direct target of miR-382. (A) Sequence alignment of miR-382 and LMO3 3'UTR. (B) Transfection of miR-382 significantly reduced LMO3 mRNA expression levels in A549 cells. (C) Transfection of miR-382 mimics significantly reduced LMO3 protein expression levels in A549 cells. (D) The luciferase activity of 293 cells cotransfected with miR-382 mimics or miR-NC mimics and LMO3 3'UTR-WT or LMO3 3'UTR-Mut was measured. miR-382 mimics reduced the luciferase activity of A549 cells transfected with LMO3 3'UTR-WT. ${ }^{\prime} \mathrm{P}<0.05$; ${ }^{* *} \mathrm{P}<0.01$. LMO3, LIM-only protein 3; miR, microRNA; UTR, untranslated region; NC, negative control; WT, wild type; mut, mutant; RT-qPCR, reverse transcription-quantitative polymerase chain reaction.

large tumor size $(\mathrm{P}=0.006)$ and lymph node metastasis $(\mathrm{P}=0.027)$. Furthermore, there was no significant difference in LMO3 expression according to sex $(\mathrm{p}=0.418)$ or age $(\mathrm{P}=0.234)$; however, high LMO3 expression was significantly associated with large tumor size $(\mathrm{P}=0.010)$, low histological grade $(\mathrm{P}=0.002)$ and lymph node metastasis $(\mathrm{P}=0.002)$.

\section{Discussion}

The expression levels of many miRNAs have previously been suggested as promising biomarkers for aiding the diagnosis, prognosis and drug-sensitivity prediction of cancer $(14,15)$. In the present study, it was demonstrated that miR-382 could inhibit cell proliferation and migration in NSCLC. In addition, it was demonstrated that LMO3, an oncogene, was a direct target of miR-382. Using LMO3 siRNA, it was also demonstrated that the anti-cancer effect of miR-382 was primarily reliant on the regulation of LMO3.

Via targeting oncogenes or tumor suppressors, deregulation of miRNAs may promote or inhibit cancer initiation and development. Notably, the role of miR-382 during cancer progression is considered controversial. In a previous study, miR-382 was demonstrated to downregulate phosphatase and tensin homolog and impacted the downstream protein kinase $\mathrm{B} /$ mechanistic target of rapamycin signaling pathway to promote angiogenesis in MKN1 human gastric cancer 

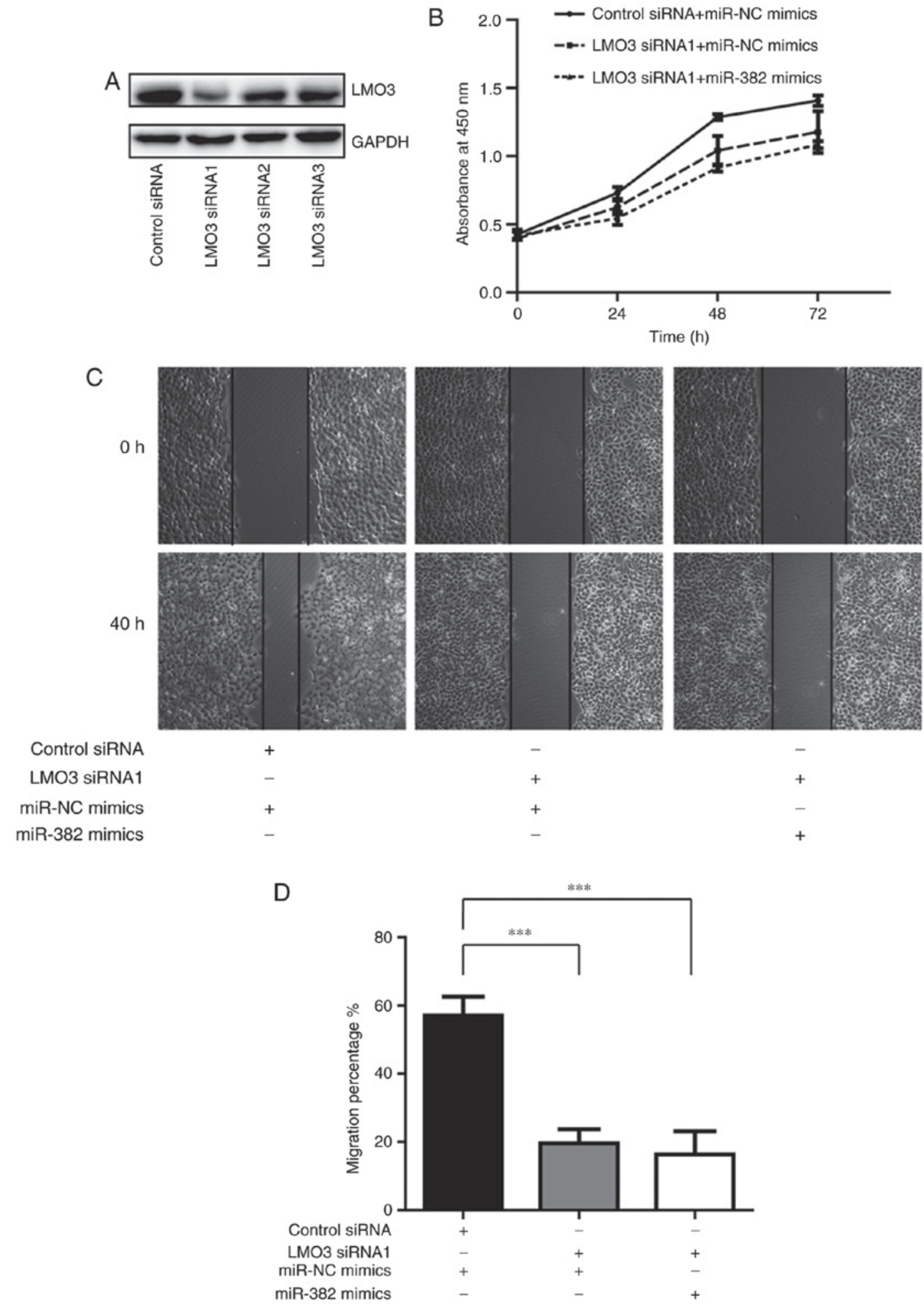

Figure 4. miR-382 suppressed A549 cell growth and migration via LMO3. (A) Transfection of all three LMO3 siRNAs successfully induced a sharp decrease in LMO3 protein expression levels in A549 cells. Compared with LMO3 siRNA2 and LMO3 siRNA3, LMO siRNA1 was the most effective siRNA in silencing LMO3. (B) Following LMO3 silencing, miR-382 mimics did not significantly alter A549 cell proliferation. (C) Following LMO3 silencing, miR-382 mimics did not significantly alter A549 cell migration ability. (D) Quantitative analysis of (C). ${ }^{* * *} \mathrm{P}<0.0001$. miR, microRNA; LMO3, LIM-only protein 3; siRNA, small interfering RNA; NC, negative control.

cells (16). However, another study indicated that miR-382 targeted tumor suppressor Ras-like, estrogen-regulated, growth inhibitor to activate the Ras/extracellular signal-regulated kinase signaling pathway and promoted cancer proliferation and metastasis in breast cancer (17). In addition, miR-382 was demonstrated to inhibit cancer cell growth in osteosarcoma (18). It was demonstrated in the present study that the miR-382 expression level was decreased in NSCLC tumor tissues and NSCLC cell lines. Furthermore, in vitro experiments indicated that miR-382 mimics greatly inhibited A549 cell growth and migration. These data were consistent with a recent study, which indicated that 
Table I. Expression of miR-382 and LMO3 in lung carcinoma patients' tissues.

\begin{tabular}{|c|c|c|c|c|c|}
\hline Factors & Patients (n) & miR-382 (fold change) & P-value & LMO3 (fold change) & P-value \\
\hline Sex & & & 0.938 & & 0.418 \\
\hline Male & 10 & $0.549 \pm 0.123$ & & $2.332 \pm 0.114$ & \\
\hline Female & 20 & $0.596 \pm 0.071$ & & $2.266 \pm 0.242$ & \\
\hline Age (years) & & & 0.331 & & 0.234 \\
\hline$<60$ & 17 & $0.610 \pm 0.084$ & & $2.454 \pm 0.183$ & \\
\hline$\geq 60$ & 13 & $0.485 \pm 0.102$ & & $2.363 \pm 0.230$ & \\
\hline Tumor size (cm) & & & 0.006 & & 0.010 \\
\hline$\geq 5$ & 16 & $0.401 \pm 0.062$ & & $2.314 \pm 0.182$ & \\
\hline$<5$ & 14 & $0.734 \pm 0.103$ & & $2.106 \pm 0.234$ & \\
\hline Histological grade & & & 0.156 & & 0.002 \\
\hline Well-intermediate differentiation & 14 & $0.473 \pm 0.063$ & & $2.243 \pm 0.150$ & \\
\hline Poor differentiation & 16 & $0.651 \pm 0.111$ & & $2.062 \pm 0.231$ & \\
\hline Lymph node metastasis & & & 0.027 & & 0.002 \\
\hline No & 22 & $0.429 \pm 0.062$ & & $2.275 \pm 0.171$ & \\
\hline Yes & 8 & $0.701 \pm 0.113$ & & $2.502 \pm 0.133$ & \\
\hline
\end{tabular}

miR, microRNA; LMO3, LIM-only protein 3.

A

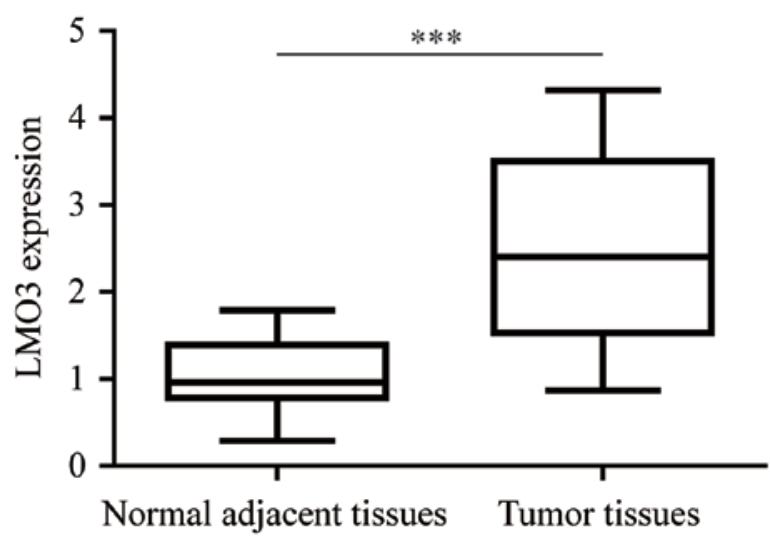

B

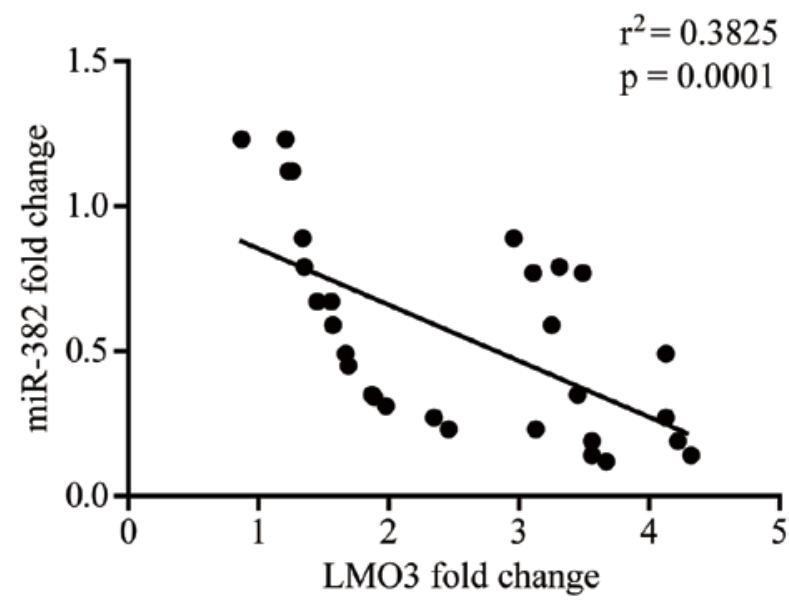

Figure 5. LMO3 expression was downregulated in tumor tissues from patients with NSCLC and negatively associated with miR-382. (A) High LMO3 mRNA expression levels were detected in tumor tissues compared with normal adjacent tissues from patients with NSCLC. (B) Correlation analysis indicated a negative association between LMO3 and miR-382 expression in tumor tissues from patients with NSCLC. ${ }^{* * *}$ P<0.0001. LMO3, LIM-only protein 3; NSCLC, non-small cell lung cancer; miR, microRNA.

miR-382 inhibits NSCLC progression via targeting SET domain-containing 5 (19).

LMO3 is a well-characterized oncogene and has been reported to be associated with many types of cancer, including NSCLC $(10,11)$. Several miRNAs have been reported to directly or indirectly regulate LMO3 to confer anti-cancer effects. For example, low levels of miR-101 have been suggested to lead to hypomethylation of the LMO3 promoter via targeting eEnhancer of zeste homolog 2, EED and DNA (cytosine-5)-methyltransferase 3A, thus contributing to LMO3 overexpression in glioma cells (20). In the present study, it was demonstrated that LMO3 was negatively associated with miR-382 in tumor tissues from patients with NSCLC.
Notably, overexpression of miR-382 decreased LMO3 mRNA and protein expression in A549 cells. Furthermore, using the luciferase assay, it was indicated that LMO3 was a direct target of miR-382. The present study also demonstrated that miR-382 mimics did not significantly enhance the inhibition of cell proliferation and migration in LMO3-silenced A549 cells. Therefore, these results suggested that miR-382 may be a tumor suppressor in NSCLC, functioning via targeting of LMO3.

In conclusion, the present findings uncovered a tumor suppressor role of miR-382 in NSCLC via directly targeting the oncogene, LMO3. These findings suggest that miR-382 may be a promising diagnostic biomarker for NSCLC. 


\section{Acknowledgements}

Not applicable.

\section{Funding}

No funding was received.

\section{Availability of data and materials}

The datasets used and/or analyzed during the current study are available from the corresponding author on reasonable request.

\section{Authors' contributions}

JZ conceived, designed and supervised the present study. JZ and DC developed the methodology, reviewed the manuscript and analyzed, and interpreted the data. DC, YZ, YL, FS, $\mathrm{ZZ}$ and $\mathrm{JZ}$ acquired the data.

\section{Ethics approval and consent to participate}

All patients provided written informed consent and the Ethics Committee of Zhangzhou Hospital Affiliated to Fujian Medical University approved the present study.

\section{Patient consent for publication}

All patients provided written informed consent.

\section{Competing interests}

The authors declare that they have no competing interests.

\section{References}

1. Jemal A, Siegel R, Ward E, Hao Y, Xu J and Thun MJ: Cancer statistics, 2009. CA Cancer J Clin 59: 225-249, 2009.

2. Toloza EM, Harpole L, Detterbeck F and McCrory DC: Invasive staging of non-small cell lung cancer: A review of the current evidence. Chest 123 (Suppl 1): S157-S166, 2003.

3. Bartel DP: MicroRNAs: Genomics, biogenesis, mechanism, and function. Cell 116: 281-297, 2004.

4. Hwang HW and Mendell JT: MicroRNAs in cell proliferation, cell death, and tumorigenesis. Br J Cancer 96 (Suppl): R40-R44, 2007.

5. Li J, Dong J, Li S, Xia W, Su X, Qin X, Chen Y, Ding H, Li H, Huang A, et al: An alternative microRNA-mediated post-transcriptional regulation of GADD 45A by p53 in human non-small-cell lung cancer cells. Sci Rep 7: 7153, 2017.
6. Ye Y, Zhuang J, Wang G, He S, Ni J, Xia W and Wang J: microRNA- 605 promotes cell proliferation, migration and invasion in non-small cell lung cancer by directly targeting LATS2. Exp Ther Med 14: 867-873, 2017.

7. Liu PL, Liu WL, Chang JM, Chen YH, Liu YP, Kuo HF, Hsieh CC, Ding YS, Chen WW and Chong IW: MicroRNA-200c inhibits epithelial-mesenchymal transition, invasion, and migration of lung cancer by targeting HMGB1. PLoS One 12: e0180844, 2017.

8. Foroni L, Boehm T, White L, Forster A, Sherrington P, Liao XB, Brannan CI, Jenkins NA, Copeland NG and Rabbitts TH: The rhombotin gene family encode related LIM-domain proteins whose differing expression suggests multiple roles in mouse development. J Mol Biol 226: 747-761, 1992.

9. Dawid IB, Breen JJ and Toyama R: LIM domains: Multiple roles as adapters and functional modifiers in protein interactions. Trends Genet 14: 156-162, 1998.

10. Aoyama M, Ozaki T, Inuzuka H, Tomotsune D, Hirato J, Okamoto Y, Tokita H, Ohira M and Nakagawara A: LMO3 interacts with neuronal transcription factor, HEN2, and acts as an oncogene in neuroblastoma. Cancer Res 65: 4587-4597, 2005.

11. Song YF, Hong JF, Liu DL, Lin QA, Lan XP and Lai GX: miR-630 targets LMO3 to regulate cell growth and metastasis in lung cancer. Am J Transl Res 7: 1271-1279, 2015.

12. Clark JR, Rumcheva P and Veness MJ: Analysis and comparison of the 7th edition American Joint Committee on Cancer (AJCC) nodal staging system for metastatic cutaneous squamous cell carcinoma of the head and neck. Ann Surg Oncol 19: 4252-4258, 2012.

13. Livak KJ and Schmittgen TD: Analysis of relative gene expression data using real-time quantitative PCR and the 2(-Delta Delta C(T)) method. Methods 25: 402-408, 2001.

14. Ma J, Lin Y,Zhan M, Mann DL, Stass SA and Jiang F: Differential miRNA expressions in peripheral blood mononuclear cells for diagnosis of lung cancer. Lab Invest 95: 1197-1206, 2015.

15. Jiang Z, Yin J, Fu W, Mo Y, Pan Y, Dai L, Huang H, Li S and Zhao J: MiRNA 17 family regulates cisplatin-resistant and metastasis by targeting TGFbetaR2 in NSCLC. PLoS One 9: e94639, 2014.

16. Seok JK, Lee SH, Kim MJ and Lee YM: MicroRNA-382 induced by HIF-1alpha is an angiogenic miR targeting the tumor suppressor phosphatase and tensin homolog. Nucleic Acids Res 42: 8062-8072, 2014.

17. Ho JY, Hsu RJ, Liu JM, Chen SC, Liao GS, Gao HW and Yu CP: MicroRNA-382-5p aggravates breast cancer progression by regulating the RERG/Ras/ERK signaling axis. Oncotarget 8: 22443-22459, 2017.

18. Xu M, Jin H, Xu CX, Sun B, Mao Z, Bi WZ and Wang Y: miR-382 inhibits tumor growth and enhance chemosensitivity in osteosarcoma. Oncotarget 5: 9472-9483, 2014.

19. Chen T, Ren H, Thakur A, Yang T, Li Y, Zhang S, Wang T and Chen M: miR-382 inhibits tumor progression by targeting SETD8 in non-small cell lung cancer. Biomed Pharmacother 86: 248-253, 2017.

20. Liu X, Lei Q, Yu Z, Xu G, Tang H, Wang W, Wang Z, Li G and Wu M: MiR-101 reverses the hypomethylation of the LMO3 promoter in glioma cells. Oncotarget 6: 7930-7943, 2015.

This work is licensed under a Creative Commons Attribution-NonCommercial-NoDerivatives 4.0 International (CC BY-NC-ND 4.0) License. 